\title{
A novel chimeric peptide binds MC3T3-E1 cells to titanium and enhances their proliferation and differentiation
}

\author{
DAN WANG, XIAOFU LIAO, XU QIN, WEI SHI and BIN ZHOU \\ Center of Stomatology, Tongji Hospital, Tongji Medical College, Huazhong University of Science and Technology, \\ Wuhan, Hubei 430030, P.R. China
}

Received November 4, 2012; Accepted February 1, 2013

DOI: $10.3892 / \mathrm{mmr} .2013 .1352$

\begin{abstract}
Previous studies have demonstrated that the modification of the titanium (Ti) surface of an implant with RGD (Arg-Gly-Asp) promotes the activity of osteoblasts. A novel Ti-binding peptide, minTBP-1, and a chimeric peptide, minTBP-1-PRGDN, have been synthesized to assist the fixing of RGD to Ti. In our previous study, minTBP-1-PRGDN demonstrated favorable affinity for Ti surfaces and facilitated the adhesion of MC3T3-E1 cells. The aim of the present study was to evaluate the effect of this chimeric peptide on the proliferation and differentiation of MC3T3-E1 cells. For this purpose, MC3T3-E1 cells were cultured and differentiation was induced on Ti discs precoated with minTBP-1-PRGDN, minTBP-1 or PRGDN. The MC3T3-E1 cells on the minTBP-1-PRGDN-precoated Ti disc were observed to exhibit the highest cell number after $24 \mathrm{~h}$ and alkaline phosphatase levels in all groups increased in a time-dependent manner. In addition, marked expression of osteogenic marker genes [osteopontin (OPN) and osteocalcin (OC)] was detected on minTBP-1-PRGDN/Ti at day 14. Mineralized deposits on minTBP-1-PRGDN/Ti presented the maximal average area and the highest number of deposits was observed on PRGDN/Ti. The present study indicates that minTBP-1-PRGDN may enhance and accelerate the activities of MC3T3-E1 cells on Ti, however, its role in vivo must be determined by further studies.
\end{abstract}

\section{Introduction}

Titanium (Ti) and its alloys have been widely used as implant materials in oromaxillofacial and other regions of bone tissue, for example, as prostheses in implantodontics and anchors in orthodontics, owing to their good biocompatibility, osseointegration, resistance to corrosion and low allergenicity $(1,2)$.

Correspondence to: Dr Bin Zhou, Center of Stomatology, Tongji Hospital, Tongji Medical College, Huazhong University of Science and Technology, 1095 Jiefang Road, Wuhan, Hubei 430030, P.R. China

E-mail: bzhou@tjh.tjmu.edu.cn

Key words: chimeric peptide, differentiation, mineralization, minTBP-1-PRGDN, proliferation, titanium
The criterion of a successful implant in vivo is osseointegration between biomaterial and bone tissue, a process in which osteoblasts are important. Various surface modifications of Ti implants have been explored to promote osseointegration and the RGD (Arg-Gly-Asp) sequence has been widely employed as a bioactive coating to improve osteoblast adhesion and proliferation, accelerate osteogenic differentiation and extracellular matrix (ECM) mineralization $(3,4)$.

The immobilization of the RGD peptide is of considerable fundamental and practical importance. Commonly, RGD covalently binds to Ti via functional moieties, including hydroxyl, amino and carboxyl radicals (5), however, this method of immobilization is associated with a number of issues, including complex treatment procedures, cytotoxity of the coupling reagent and deactivation of the active group rapidly by hydrolysis (6). Previously, peptide aptamers (i.e., binders) which interact with inorganic materials have been artificially created and used as a 'glue' to link various biomolecules to the surface of inorganic metals (7). TBP-1 (RKLPDAPGMHTW), a novel peptide aptamer isolated from a linear 12-mer peptide phage library, has been confirmed to be capable of specifically recognizing and interacting with $\mathrm{Ti}$, by its $\mathrm{N}$-terminal sequence, RKLPDA (minTBP-1) (8). In our previous study, a novel chimeric peptide RKLPDAPRGDN (minTBP-1-PRGDN) was synthesized by connecting minTBP-1 to the N-terminal of PRGDN. This chimeric peptide was found to exhibit favorable affinity for the Ti surface and the modified Ti was associated with improved attachment and spreading of MC3T3-E1 cells (9).

The MC3T3-E1 cell, derived from neonatal mouse calvaria, has been widely used as a model osteoprogenitor cell in bone tissue engineering (10). MC3T3-E1 cells on implant surfaces are known to undergo three phases: initial adhesion (from minutes to hours), proliferation (days) and differatiation (weeks to months) (11). In the present study, the biological behavior of MC3T3-E1 cells following adhesion to minTBP-1-PRGDN modified Ti discs was further investigated. Cell proliferation and differentiation were estimated by cell numbers, relative gene expression and alkaline phosphatase activity (ALP). In addition, ECM mineralization was assessed.

\section{Materials and methods}

Preparation of Ti discs and peptides. Commercially pure Ti discs (diameter, $15 \mathrm{~mm}$; thickness, $1 \mathrm{~mm}$; Cp Ti; Baoji 
Nonferrous Metal Industry Co., Baoji, China) were polished, cleaned, oxidized and sterilized as described previously (12). The chimeric peptide RKLPDAPRGDN (minTBP-1-PRGDN; $1,238.38 \mathrm{~g} / \mathrm{mol}$ ) was synthesized by connecting RKLPDA (minTBP-1) to the N-terminal of PRGDN (derived from the consecutive sequence of human BSP, GenBank accession no. AAA60549.1, residues 285-289). RKLPDA (698.83 g/mol), PRGDN (557.57 g/mol) and RKLPDAPRGDN were all synthesized by ChinaPeptides Co., Ltd. (Shanghai, China). All peptides were synthesized using the solid phase peptide synthesis method, purified by HPLC and identified by amino acid analysis. The lyophilized peptides were reconstituted in sterile deionized water to a final concentration of $100 \mu \mathrm{g} / \mathrm{ml}$.

Ti disc coating. Sterilized Ti discs were placed at the bottom of 24-well culture plates which had the same diameter as the $\mathrm{Ti}$ disc to prevent the non-specific binding of cells to the bottom of the culture plate. The discs were then divided into 4 groups and were coated with minTBP-1-PRGDN/Ti, minTBP-1/Ti or PRGDN/Ti overnight at $4^{\circ} \mathrm{C}$, or were uncoated (control/Ti). Prior to cell seeding, all discs were washed several times with phosphate-buffered saline (PBS; Gibco-BRL, Carlsbad, CA, USA) to remove unbound peptides and pre-warmed in an incubator at $37^{\circ} \mathrm{C}$.

MC3T3-E1 cell culture. Mouse osteoblastic MC3T3-E1 cells were cultured as described previously (9).

MC3T3-E1 cell proliferation. To investigate cell proliferation, MC3T3-E1 cells were cultured onto Ti discs at a density of $2 \times 10^{4}$ cells $/ \mathrm{cm}^{2}$ in 24 -well tissue culture plates and incubated in medium containing $10 \%$ fetal bovine serum for 24,48 and $72 \mathrm{~h}$. Following each incubation period, quantification of proliferated cells was performed by colorimetry using alamarBlue (Biosource International, Inc., Camarillo, CA, USA). Briefly, medium was aspirated and proliferative cells were incubated in serum-free culture medium containing $10 \%$ $(\mathrm{v} / \mathrm{v})$ fresh alamarBlue for $5 \mathrm{~h}$. Cell number was measured as the difference in absorbance at 570 and $600 \mathrm{~nm}$ by a microplate spectrophotometer ( $\mu$ Quant; BioTek Instruments, Inc., Winooski, VT, USA).

MC3T3-E1 cell differentiation. For ALP activity, osteogenic marker gene expression and matrix mineralization assays, MC3T3-E1 cells were seeded onto the various modified Ti surfaces at a density of $1 \times 10^{4}$ cells $/ \mathrm{cm}^{2}$ in 24 -well tissue culture plates. After $24 \mathrm{~h}$, the cells were cultured in osteogenic induction medium, which contained $50 \mathrm{mg} / \mathrm{l}$ ascorbic acid, $10 \mathrm{mmol} / \mathrm{l} \beta$-glycerophosphate and $10^{-8} \mathrm{~mol} / \mathrm{l}$ hexadecadrol. The medium was replaced every $48 \mathrm{~h}$.

ALP activity assay. ALP activity was determined by a colorimetric endpoint assay, in which the enzymatic conversion of p-nitrophenyl phosphate (pNPP; N9389; Sigma-Aldrich, St. Louis, MO, USA) to the yellowish product, p-nitrophenol (pNP), was measured. Following osteogenic induction for 28 days, cells on Ti discs were rinsed with PBS, detached with $0.25 \%(w / v)$ trypsin and then collected with $1 \%$ Triton $\mathrm{X}-100$ buffer solution ( $\mathrm{pH} 7.6$ ) containing $1 \mathrm{mM} \mathrm{MgCl} \cdot 6 \mathrm{H}_{2} \mathrm{O}$ and $50 \mathrm{mM}$ Tris. Following treatment with hypersound on ice for
$30 \mathrm{~min}$, an aliquot $(20 \mu \mathrm{l})$ of the cell lysate was incubated with $1 \mathrm{ml}$ reaction solution (containing $1 \mathrm{mM} \mathrm{MgCl}, 1 \mathrm{mM} \mathrm{ZnCl} \mathrm{Zn}_{2}$ and $1 \mathrm{mg} / \mathrm{ml} \mathrm{pNPP}$ ) in the dark for $30 \mathrm{~min}$ at room temperature. To terminate the enzymatic conversion of pNPP to pNP in the presence of ALP, $3 \mathrm{~N} \mathrm{NaOH}$ was added, followed by incubation for an additional $5 \mathrm{~min}$. ALP activity was determined by spectrophotometrical quantification of pNP at $405 \mathrm{~nm}$ using an ELISA reader (model 550; Bio-Rad, Hercules, CA, USA). A bicinchoninic acid protein assay reagent (\#23227; Pierce Biotechnology, Inc., Rockford, IL, USA) was used to determine the total intracellular protein content. ALP activity was normalized to $\mathrm{U} \mathrm{pNP} / \mathrm{mg}$ protein.

Quantitative real-time polymerase chain reaction ( $q$-RT-PCR). At the end of each osteogenic induction (7, 14 and 21 days), total RNA was isolated with an RNeasy kit (Qiagen, Tokyo, Japan) according to the manufacturer's instructions. Reverse transcription was performed following standard procedures with SuperScript Reverse Transcriptase (Invitrogen Life Technologies, Carlsbad, CA, USA). SYBR-Green-based q-RT-PCR amplifications were performed using the following primer sets: osteopontin (OPN), 5'-CTT TCA CTC CAA TCG TCC CTA C-3' and 5'-CTG CCC TTT CCG TTG TTG TC-3' $\left(58^{\circ} \mathrm{C}, 238 \mathrm{bp}\right)$; osteocalcin (OC), 5'-TCT CTG CTC ACT CTG CTG GC-3' and 5'-GGG ACT GAG GCT CCA AGG TA-3' $\left(60^{\circ} \mathrm{C}, 155 \mathrm{bp}\right)$; and $\beta$-actin, 5'-GTG GGC CGC TCT AGG CAC CAA-3' and 5'-CTC TTT GAT GTC ACG CAC GAT TTC-3' (58 $\left.{ }^{\circ} \mathrm{C}, 249 \mathrm{bp}\right)$. Triplicate reactions were performed for each sample. Expression values of each sample were normalized against $\beta$-actin expression levels.

Alizarin red-S $(A R-S)$ staining and analysis of mineralized deposits by metallurgical microscopy. Following the 28-day osteogenic induction period, cells were fixed with $4 \%$ paraformaldehyde in PBS for $15 \mathrm{~min}$ at $4^{\circ} \mathrm{C}$ and rinsed with PBS. Fixed cells were stained for 10 min with $0.1 \%$ AR-S (A5533; Sigma-Aldrich) at room temperature with gentle rotation and washed with PBS. For each group, three visual fields of the same magnification (x100) were randomly selected and images were captured using a metallurgical microscope (Axiovert 200MAT; Carl Zeiss Light Microscopy, Göttingen, Germany). Image-Pro ${ }^{\circledR}$ Plus software package (6.0.0 edition, Media Cybernetics, Inc., Rockville, MD, USA) was employed for image analysis. Briefly, the mineralized deposits in each group were counted and their relative sizes were quantified by measuring the average number of pixels captured using the Image-Pro ${ }^{\circledR}$ Plus software package.

Statistical analysis. All experiments were performed at least in triplicate. Results were analyzed by Student's t-test between 2 groups of experiments and one-way ANOVA in $>2$ groups of experiments. If the ANOVA test identified a significant difference, further post hoc Fisher's LSD multiple comparison test was applied. Data are presented as mean \pm SD (bars). $\mathrm{P}<0.05$ was considered to indicate a statistically significant difference.

\section{Results}

MC3T3-E1 cell proliferation. The alamarBlue assay was used to determine relative cell proliferation levels at 24,48 and $72 \mathrm{~h}$. 


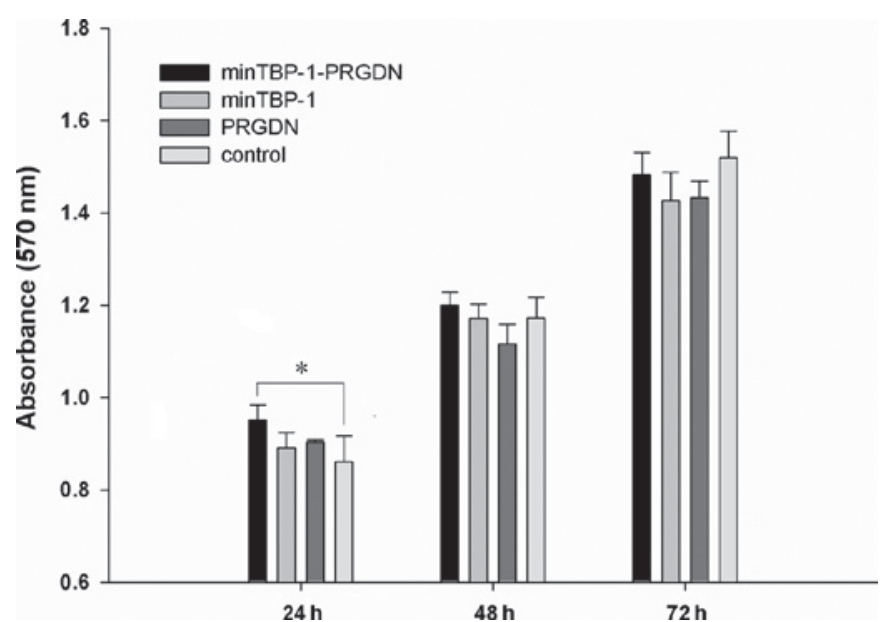

Figure 1. Quantification of proliferation of MC3T3-E1 cells by analysis of absorbance of alamarBlue. Proliferation on minTBP-1-PRGDN/Ti was observed to be significantly greater than that on control/Ti after $24 \mathrm{~h}\left({ }^{*} \mathrm{P}<0.05\right)$. Data are presented as the mean $\pm \mathrm{SD}$ ( $\mathrm{n}=3$, each group). Ti, titanium.

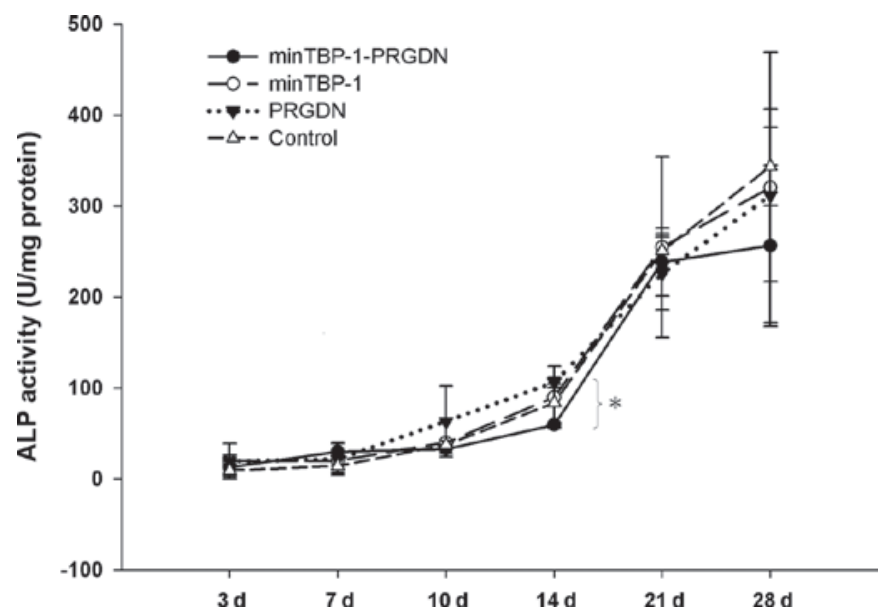

Figure 2. ALP activity of MC3T3-E1 cells. On day 14, cells on control/T exhibited higher ALP activity than that on minTBP-1-PRGDN/Ti ( $\mathrm{P}<0.05$ ). ALP activity was normalized against units $\mathrm{pNP} / \mathrm{mg}$ protein. Data are presented as the mean $\pm \mathrm{SD}$ ( $\mathrm{n}=3$, each group). ALP, alkaline phosphatase; Ti, titanium.

As demonstrated in Fig. 1, increased absorbance was observed in all groups at the end of the culture period indicating that MC3T3-E1 cell proliferation occurred in all 4 groups. In particular, the proliferation on minTBP-1-PRGDN/Ti was observed to be significantly greater than that on control/Ti after $24 \mathrm{~h}(\mathrm{P}<0.05)$.

ALP activity assay. For each group, ALP activity was at a low basal level on day 3 and increased steadily until day 14, this was followed by a rapid increase which peaked on day 28 (Fig. 2). These observations indicate that MC3T3-E1 cells in all groups differentiated towards mature osteoblasts. Of note, the ALP activity of cells on control/Ti was much higher than those on minTBP-1-PRGDN/Ti on day $14(\mathrm{P}<0.05)$.

Expression of osteogenic marker genes. Gene expression profiling data derived from q-RT-PCR for osteogenic markers were assessed and are shown in Fig. 3. Cells on
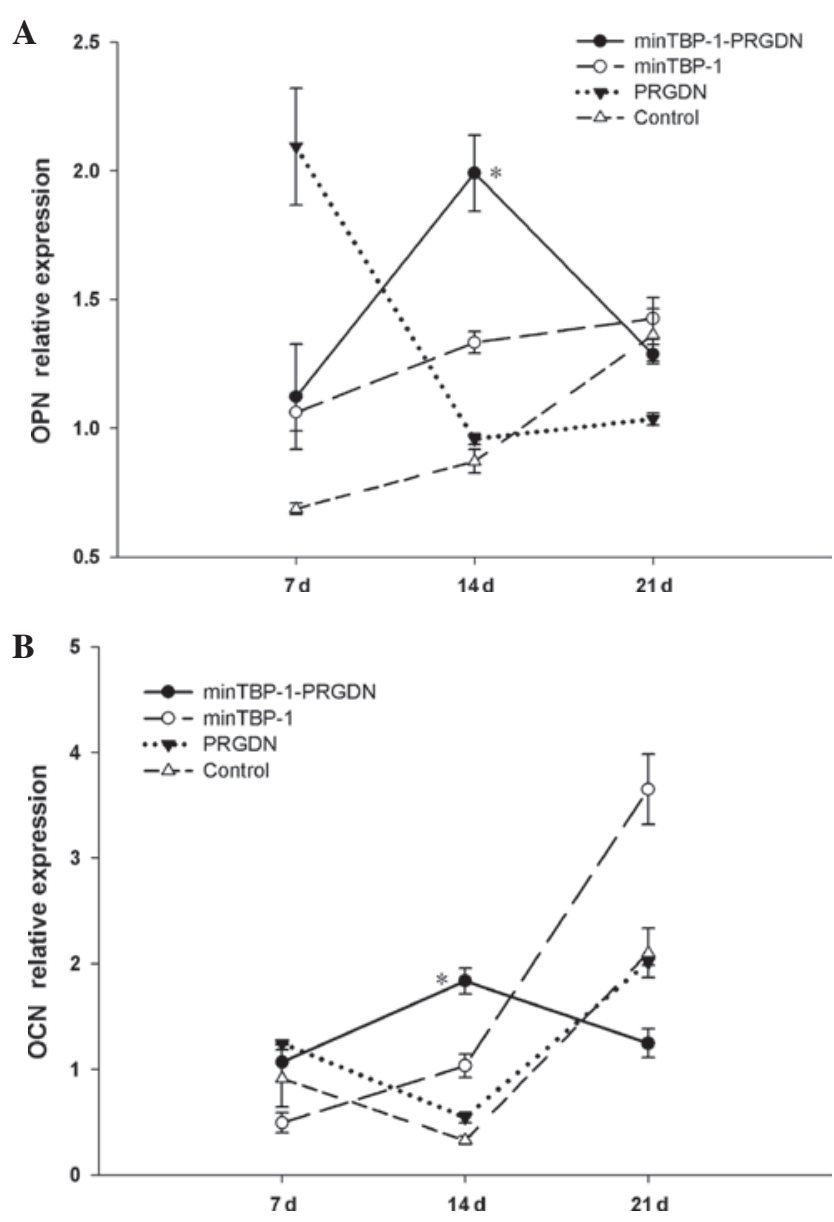

Figure 3. Mean mRNA expression levels of osteogenic marker genes of MC3T3-E1 cells by q-RT-PCR. Cells on minTBP-1-PRGDN/Ti were found to express OC and OPN genes at the highest levels following osteogenic induction for 14 days $(" \mathrm{P}<0.05)$. Data are presented as the mean $\pm \mathrm{SD}(\mathrm{n}=3$, each group). qRT-PCR, quantitative real-time polymerase chain reaction; $\mathrm{Ti}$, titanium; OPN, osteopontin; OC, osteocalcin.

minTBP-1-PRGDN/Ti were found to express the highest levels of OPN (Fig. 3A) and OC (Fig. 3B) mRNA after 14 days of osteogenic induction $(\mathrm{P}<0.05)$.

Mineralized deposits. On day 28, thin spherical deposits, which were stained red by AR-S, were distributed uniformly on the Ti discs in all groups (Fig. 4). The average area of mineralized deposits was highest on minTBP-1-PRGDN/Ti and lowest on minTBP-1/Ti and the difference between these two groups was significant $(\mathrm{P}<0.05$; Fig. 5A). The average number of mineralization deposits on PRGDN/Ti was the highest and was significantly higher than the numbers on minTBP-1/Ti and control/Ti ( $\mathrm{P}<0.05$; Fig. 5B).

\section{Discussion}

To assistant RGD peptide immobilization on the Ti surface, the chimeric peptide minTBP-1-PRGDN was previously designed and synthesized with the aim of achieving a double binding function by minTBP-1 specifically recognizing and binding Ti and PRGDN associating with integrin receptors on the osteoblast membrane (9). In our previous study, the chimeric peptide was found to exhibit an affinity for $\mathrm{Ti}$ and the ability 
A

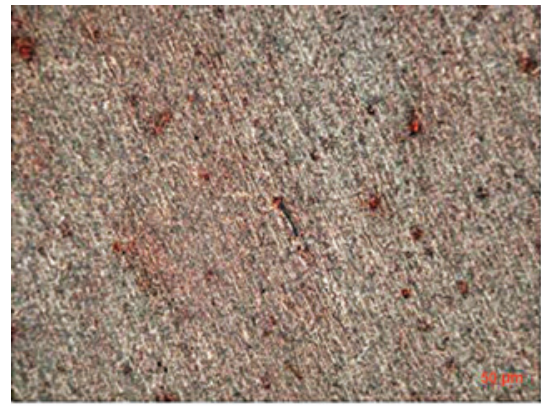

C

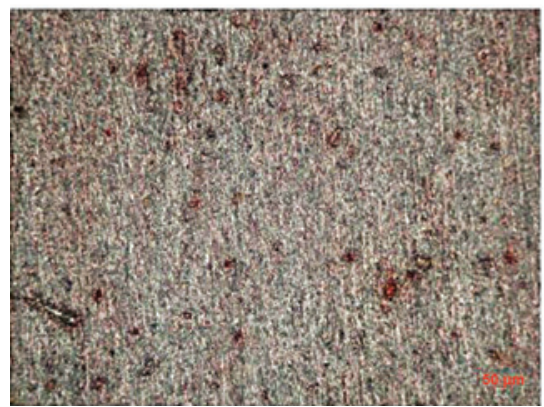

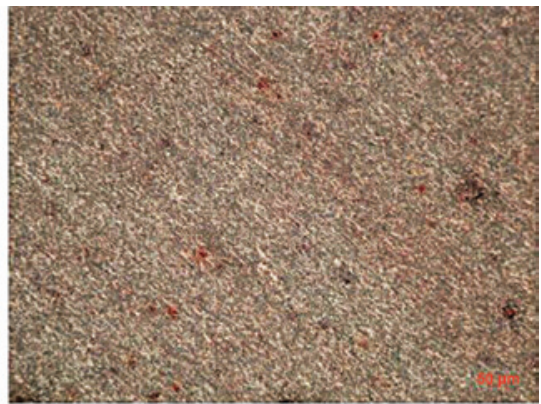

D

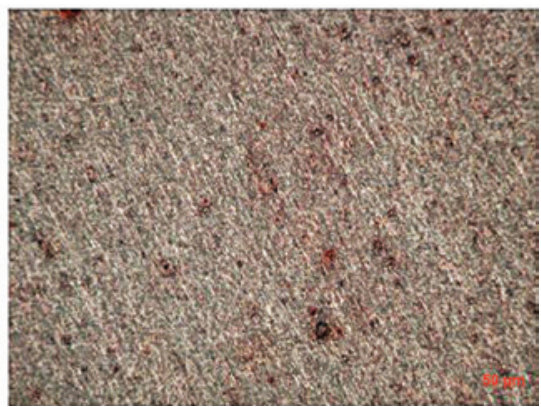

Figure 4. Alizarin red-S staining of MC3T3-E1 cells on minTBP-1-PRGDN/Ti, minTBP-1/Ti, PRGDN/Ti and control/Ti following 28 days of osteogenic induction. Mineralized deposits were stained red. Images were captured by metallurgical microscope (magnification, $\mathrm{x} 100 ;$ bar=50 $\mu \mathrm{m}$ ). Ti, titanium.

A

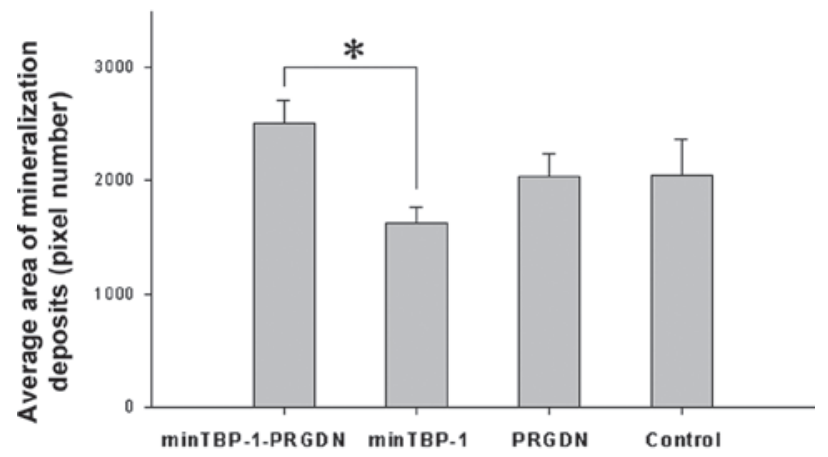

B

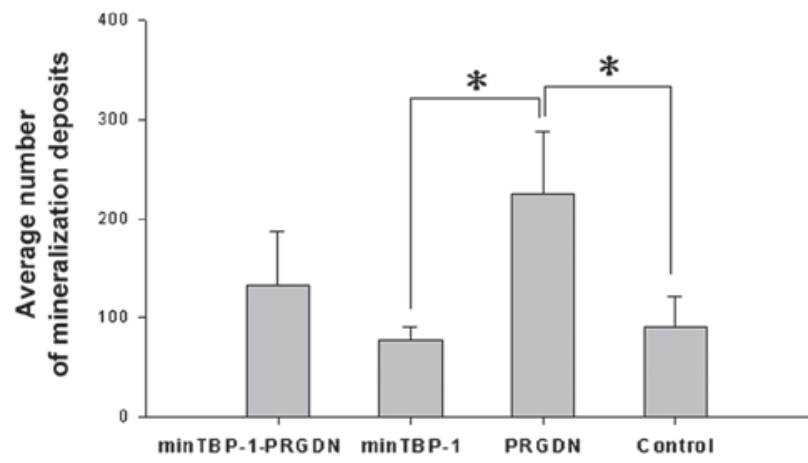

Figure 5. Average area/number of mineralized deposits of MC3T3-E1 cells following 28 days of osteogenic induction analyzed by Image-Pro ${ }^{\circledR}$ Plus software. Data are presented as the mean $\pm \mathrm{SD}$ ( $\mathrm{n}=3$, each group). (A) Average area of mineralized deposits by pixel number. (B) Average number of mineralized deposits. $(\mathrm{P}<0.05)$.

to facilitate the adhesion of MC3T3-E1 cells. In the present study, the effect of minTBP-1-PRGDN on the bioactivities of MC3T3-E1 cells following adhesion was explored.

Favorable adhesion and fast proliferation has been demonstrated to aggregate osteoblasts into multilayers which provide collagen support as the basis of mineralized deposit formation (13). In the present study, cell numbers increased with time in all groups, which indicated that MC3T3-E1 cells proliferated well. In particular, minTBP-1-PRGDN rapidly accelerated the proliferation of the cells (at $24 \mathrm{~h}$ ), indicating that this chimeric peptide may favor the differentiation of MC3T3-E1 cells.

ALP represents a standard marker of ECM development and maturation for in vitro experiments and is expressed from the early stages of osteoblast differentiation, decreases slightly when mineral deposition occurs and is then maintained at relatively high levels (14). In the present study, ALP activity increased persistently with time in all groups. and did not decrease. This result is consistent with the findings of Oya et al that the ALP activity of MC3T3 E1 cells kept increasing with time from the beginning of osteogenic induction to day 28 (15). Elevated ALP activity is an indicator of improved MC3T3-E1 cell function and matrix production. In the present study, cells on PRGDNT/Ti exhibited a marked increase in ALP activity by day 10 and 14. This observation is not considered to be unusual since RGD, a common element of the majority of ECM proteins, has been reported to advance the ALP activity induced by RGD-ligand binding to osteoblast integrin receptors in a number of studies $(16,17)$.

OPN and OC, secreted by differentiated osteoblasts, are the main noncollagen proteins and participate in ECM mineralization. In the current study, OPN and OC rapidly peaked on minTBP-1-PRGDN/Ti in the middle stage of differentiation (day 14). It should be noted that the middle stage of osteoblast differentiation is extremely important as, during this stage, the ECM matures gradually and mineralized deposits begin to form (18). The results of the current study indicate that the chimeric peptide, minTBP-1-PRGDN, accelerated MC3T3-E1 cell osteogenic differentiation, which is likely to promote the osseointegration of modified Ti implants in vivo.

Mineralization of the ECM is a late marker of osteoblast differentiation and an important indicator of the osteoinductive ability of implant biomaterial (19). During mineralization, 
amorphous $\mathrm{Ca} / \mathrm{P}$ materials are deposited extracellularly and propagate into the collagen fibril matrix, leading to formation of mineralized deposits (18). In the present study, the average area of mineralized deposits on minTBP-1-PRGDN/Ti was the highest at the end of osteogenic induction (day 28). We hypothesize that the chimeric peptide facilitated the accumulation of hydroxyapatite on the modified Ti surface. Of note, MC3T3-E1 cell attachment to minTBP-1-PRGDN/Ti was the most evident (9) and the cell proliferation in the minTBP-1-PRGDN/ Ti group was higher than that in the other groups in the initial $24 \mathrm{~h}$. In addition, this chimeric peptide contributed to the expression of mineralization-related genes (OPN/OC) in the middle stage of MC3T3-E1 cell differentiation. Collectively, these observations are consistent. We hypothesize that increased attachment and active movement facilitated the rapid confluency of MC3T3-E1 cells on minTBP-1-PRGDN/Ti, which contributed to the rapid proliferation, differentiation and mineralization of the ECM. On day 14, ALP activity of MC3T3-E1 cells on PRGDN/Ti was the most conspicuous in this study, which may contribute to the highest mineralized deposit number on the PRGDN/Ti surface. This result was consistent with reports that RGD-containing coatings may promote osteoblast activities and mineralization on biomaterials $(3,4)$.

In conclusion, the present study indicated that the chimeric peptide minTBP-1-PRGDN facilitated the biological behaviour of MC3T3-E1 cells, to some extent, following adhesion to the Ti surface and mineralization of the ECM. This study may provide an important insight into the value of the biomimetic coating of Ti implants and the application of functional adhesion peptides which may facilitate bone remodeling. Further in vivo studies are required to fully understand the role of minTBP-1-PRGDN in osseointegration between Ti implants and bone tissue and this investigation is currently in progress.

\section{Acknowledgements}

This study was supported by a grant from the National Science Foundation of China (No. 81171450).

\section{References}

1. Long M and Rack HJ: Titanium alloys in total joint replacement - a materials science perspective. Biomaterials 19: 1621-1639, 1998.

2. Liu XY, Chu PK and Ding CX: Surface modification of titanium, titanium alloys and related materials for biomedical applications. Mater Sci Eng R 47: 49-121, 2004.

3. Ferris DM, Moodie GD, Dimond PM, Gioranni CW, Ehrlich MG and Valentini RF: RGD-coated titanium implants stimulate increased bone formation in vivo. Biomaterials 20: 2323-2331, 1999.
4. Cavalcanti-Adam EA, Shapiro IM, Composto RJ, Macarak EJ and Adams CS: RGD peptides immobilized on a mechanically deformable surface promote osteoblast differentiation. J Bone Miner Res 17: 2130-2140, 2002.

5. Sawyer AA, Weeks DM, Kelpke SS, McCracken MS and Bellis SL: The effect of the addition of a polyglutamate motif to RGD on peptide tethering to hydroxyapatite and the promotion of mesenchymal stem cell adhesion. Biomaterials 26: 7046-7056, 2005.

6. Hersel U, Dahmen C and Kessler H: RGD modified polymers: biomaterials for stimulated cell adhesion and beyond. Biomaterials 24: 4385-4415, 2003.

7. Baneyx F and Schwartz DT: Selection and analysis of solid-binding peptides. Curr Opin Biotechnol 18: 312-317, 2007.

8. Sano K and Shiba K: A hexapeptide motif that electrostatically binds to the surface of titanium. J Am Chem Soc 125: 14234-14235, 2003.

9. Wang D, Mao J, Zhou B, Liao XF, Gong SQ, Liu Y and Zhang JT: A chimeric peptide that binds to titanium and mediates MC3T3-E1 cell adhesion. Biotechnol Lett 33: 191-197, 2011.

10. Sudo H, Kodama HA, Amagai Y, Yamamoto S and Kasai S: In vitro differentiation and calcification in a new clonal osteogenic cell line derived from new born mouse calvaria. J Cell Biol 96: 191-198, 1983.

11. Quarles LD, Yohay DA, Lever LW, Caton R and Wenstrup RJ: Distinct proliferative and differentiated stages of murine MC3T3-E1 cells in culture: An in vitro model of osteoblast development. J Bone Miner Res 7: 683-692, 1992.

12. Liu Y, Mao J, Zhou B, Wei W and Gong S: Peptide aptamers against titanium-based implants identified through phage display. J Mater Sci Mater Med 21: 1103-1107, 2010.

13. Pockwinse SM, Stein JL, Lian JB and Stein GS: Developmental stage-specific cellular responses to vitamin D and glucocorticoids during differentiation of the osteoblast phenotype: interrelationship of morphology and gene expression by in situ hybridization. Exp Cell Res 216: 244-260, 1995.

14. Choi JY, Lee BH, Song KB, Park RW, Kim IS, Sohn KY, Jo JS and Ryoo HM: Expression patterns of bone-related proteins during osteoblastic differentiation in MC3T3-E1 cells. J Cell Biochem 61: 609-618, 1996.

15. Oya K, Tanaka Y, Saito H, Kurashima K, Nogi K, Tsutsumi H, Tsutsumi Y, Doi H, Nomura N and Hanawa T: Calcification by MC3T3-E1 cells on RGD peptide immobilized on titanium through electrodeposited PEG. Biomaterials 30: 1281-1286, 2009.

16. Ruoslahti E: RGD and other recognition sequences for integrins. Annu Rev Cell Dev Biol 12: 697-715, 1996.

17. Sawyer AA, Hennessy KM and Bellis SL: The effect of adsorbed serum proteins, RGD and proteoglycan-binding peptides on the adhesion of mesenchymal stem cells to hydroxyapatite. Biomaterials 28: 383-392, 2007.

18. Rohde $\mathrm{M}$ and Mayer H: Exocytotic process as a novel model for mineralization by osteoblasts in vitro and in vivo determined by electron microscopic analysis. Calcif Tissue Int 80: 323-336, 2007.

19. Declercq HA, Verbeeck RM, De Ridder LI, Schacht EH and Cornelissen MJ: Calcification as an indicator of osteoinductive capacity of biomaterials in osteoblastic cell cultures. Biomaterials 26: 4964-4974, 2005. 